\title{
Strained-Si single-gate versus unstrained-Si double-gate MOSFETs
}

\author{
F M Bufler, A Schenk and W Fichtner \\ Institut für Integrierte Systeme, ETH Zürich, Gloriastrasse 35, CH-8092 Zürich, Switzerland \\ E-mail: bufler@iis.ee.ethz.ch
}

Received 28 July 2003

Published 3 March 2004

Online at stacks.iop.org/SST/19/S122 (DOI: 10.1088/0268-1242/19/4/043)

\begin{abstract}
Self-consistent full-band Monte Carlo simulations are employed to compare the performance of nanoscale strained-Si single-gate (SG) and unstrained-Si double-gate (DG) MOSFETs for a gate length of $25 \mathrm{~nm}$. Almost the same on-current as in the DG-MOSFET can be achieved by strain in a

SG-MOSFET for the same gate overdrive. This is due to the compensation of the higher electron sheet density in the two inversion channels of the DG-MOSFET by the higher strain-enhanced velocity in the channel of the SG-MOSFET. The on-current of the strained-Si SG-MOSFET is almost $10 \%$ larger for a channel orientation along the crystallographic $\langle 100\rangle$ direction than for the $\langle 110\rangle$ direction. This confirms that the on-current is determined by quasi-ballistic transport, because the maximum enhancement of the in-plane velocity in bulk (001)-strained $\mathrm{Si}$ in the $\langle 100\rangle$ direction is 5\% at medium electric fields (the low-field mobilities and saturation velocities are the same and the difference in the thermal injection velocities is negligibly small), whereas the transient bulk velocity overshoot peak is $30 \%$ larger in the $\langle 100\rangle$ direction.
\end{abstract}

\section{Introduction}

As the performance enhancement of silicon microelectronics by scaling of conventional bulk MOSFETs is thought to approach its physical and technological limits, alternative concepts are currently being explored. Double-gate (DG) and strained-Si MOSFETs are considered as the two most promising candidates which may take us to the limit of silicon complementary metal-oxide-semiconductor (CMOS) technology [1,2] and high performance of such structures has been confirmed experimentally [3, 4]. At the same time, these nanoscale devices are interesting from a physical point of view because their on-state is governed by quasi-ballistic transport which is beyond the classical drift-diffusion (DD) equations. It is the aim of this paper to compare via self-consistent fullband Monte Carlo (FBMC) simulation [5] DG and strained-Si MOSFETs in an extremely scaled prospective structure and to explore the physical mechanisms which influence the oncurrent in the quasi-ballistic transport regime.

\section{Monte Carlo model and device structures}

The investigations in this paper are performed using the self-consistent full-band Monte Carlo simulator SPARTA [5].
The band structures are obtained from nonlocal empirical pseudopotential calculations [6] where in addition the spinorbit interaction has also been taken into account. The scattering mechanisms comprise three $f$-type and three $g$-type intervalley phonon processes with exactly the same coupling constants as used by Jacoboni and Reggiani [7], intravalley phonon scattering, impact ionization, a calibrated Ridley model for impurity scattering and surface roughness scattering consisting of a combination of specular and diffusive scattering [8] with a percentage of $15 \%$ diffusive scattering. The biaxial tensile strain caused by the lattice mismatch between a silicon layer and a SiGe substrate shifts four of the six valleys upwards in energy leading to a smaller in-plane conductivity mass and reduced intervalley scattering. Experiments [9] and theoretical works [10-13] report a strongly improved bulk low-field mobility in strained Si and also measurements [4] as well as Monte Carlo simulations [14] of nanoscale bulk n-MOSFETs find a pronounced enhancement of the on-current under strain.

The unstrained-Si DG MOSFET has a gate length of $L_{\mathrm{G}}=25 \mathrm{~nm}$, a film thickness of $t_{\mathrm{Si}}=6.25 \mathrm{~nm}$, an oxide thickness of $t_{\mathrm{OX}}=1 \mathrm{~nm}$ and a tungsten metal gate. The doping level in the source/drain regions is $10^{20} \mathrm{~cm}^{-3}$ and falls off into the undoped channel by $2.5 \mathrm{~nm} \mathrm{dec}^{-1}$. In the single-gate 
Strained-Si single-gate versus unstrained-Si double-gate MOSFETs

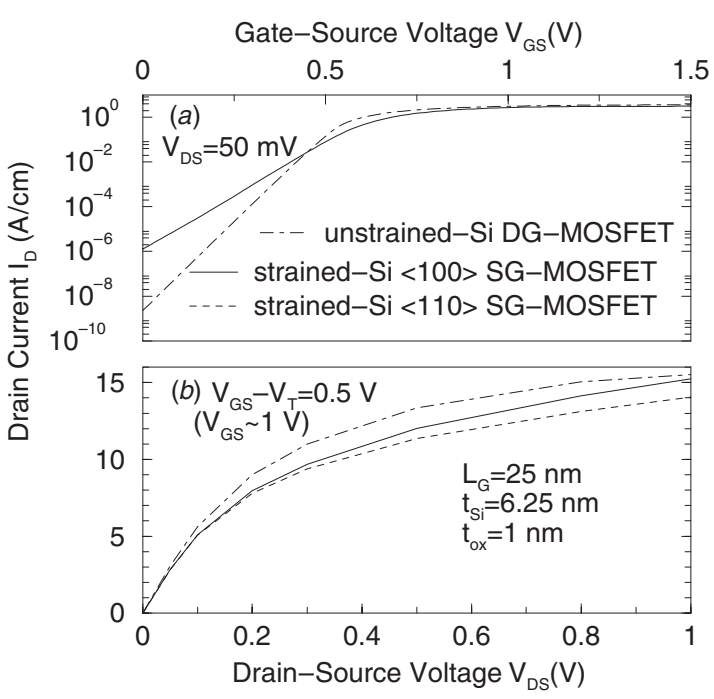

Figure 1. Transfer characteristics according to the DD model (a) and output characteristics computed by FBMC simulation (b) of an unstrained-Si DG-MOSFET and a strained-Si SG-MOSFET with a channel oriented along the crystallographic in-plane $\langle 110\rangle$ or $\langle 100\rangle$ direction. Note that there is no difference between the $\langle 110\rangle$ and the $\langle 100\rangle$ directions in the strained-Si transfer characteristics at $V_{\mathrm{DS}}=$ $50 \mathrm{mV}(a)$, because the anisotropy only appears at higher drain voltages $($ see $(b))$.

(SG) strained-Si SiGe-on-insulator (strained-SOI) structure, the silicon channel is grown on a $100 \mathrm{~nm}$ p-doped relaxed SiGe buffer layer which is on top of a $50 \mathrm{~nm}$ buried oxide. Such a structure can be obtained by the combination of separationby-implanted-oxygen (SIMOX) technology with a Si regrowth technique found suitable for device fabrication [15]. The germanium content of the relaxed SiGe buffer is $40 \%$ in this simulation study.

\section{Simulation results}

Figure 1(a) shows the transfer characteristics where the Ge content and the doping level in the SiGe buffer were adjusted to yield, similar to the DG MOSFET, a threshold voltage of about $V_{\mathrm{T}} \sim 0.5 \mathrm{~V}$. The off-current in the strained-Si MOSFET is about three orders of magnitude larger than in the DG MOSFET, but the value of $0.1 \mathrm{nA} \mu \mathrm{m}^{-1}$ is still very small and appropriate for high-speed applications. The output characteristics in figure $1(b)$, computed for the same gate overdrive of $V_{\mathrm{GS}}-V_{\mathrm{T}}=0.5 \mathrm{~V}$, demonstrate that almost the same on-current $I_{\text {on }}$ as in the DG MOSFET can be achieved by strain in a SG MOSFET. The reason can be deduced from figure 2 where the profiles of sheet density and drift velocity are displayed: the higher charge density of the two inversion channels in the DG MOSFET is compensated by a higher drift velocity in strained $\mathrm{Si}$.

The physically striking effect is that $I_{\text {on }}$ of the strainedSi MOSFET is almost $10 \%$ larger for a $\langle 100\rangle$ than for a $\langle 110\rangle$ orientation of the channel in view of equal low-field mobilities $\mu_{\text {low }}$ and saturation velocities for both directions with a maximum enhancement in the $\langle 100\rangle$ direction of $5 \%$ at $25 \mathrm{kV} \mathrm{cm}^{-1}$ (and negligible differences in the thermal injection velocities $v_{\text {inj }}$ ). This direction dependence of $I_{\text {on }}$ in strained $\mathrm{Si}$ is similar to that found in nanoscale bulk n-MOSFETs in
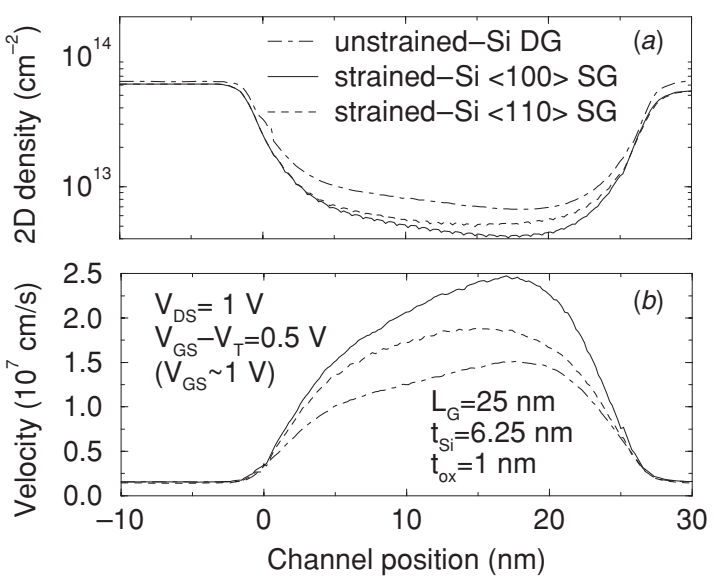

Figure 2. Profiles along the channel of (a) the sheet density obtained by integration of the electron density perpendicular to the $\mathrm{Si} / \mathrm{SiO}_{2}$ interface over the silicon film thickness $t_{\mathrm{Si}}$ and $(b)$ the drift velocity, averaged perpendicularly to the interface with the electron density, in the on-state of the three device configurations of figure 1.

[14] where also the bulk simulation results for strained and unstrained Si are shown. This confirms that (i) quasi-ballistic transport determines $I_{\mathrm{on}}$, since the transient bulk overshoot peak is $30 \%$ larger for the $\langle 100\rangle$ direction in strained Si because of a stronger band curvature above $100 \mathrm{meV}$ [16], and (ii) widely used models ascribing $I_{\text {on }}$ to $v_{\text {inj }}$ and $\mu_{\text {low }}$-dependent backscattering [17] fail to describe the anisotropy of $I_{\text {on }}$ in strained Si. The increasing contribution of quasi-ballistic transport to $I_{\mathrm{on}}$ in strained $\mathrm{Si}$ is also the explanation why strain still improves $I_{\mathrm{on}}$ for decreasing gate lengths. The apparent discrepancy as pointed out in [18] was that smaller gate lengths involve higher longitudinal electric fields, but that the oncurrent is still improved by strain in nanoscale MOSFETs [4] despite a vanishing strain-induced velocity enhancement in the high-field limit. However, the electrons are subject to quasi-ballistic overshoot effects when experiencing a strong field upon entering the source side of the channel and these overshoot effects are stronger in strained $\mathrm{Si}$. This can also be seen in figure $2(b)$ which shows that the electrons travel in strained Si almost immediately after entering the channel with a velocity above the saturation velocity (see also [14] for a more comprehensive discussion).

In conclusion, our simulation study shows a similar high potential of both DG and strained-Si MOSFETs for the nanoscale regime. To decide which of the two approaches is to be preferred will require more detailed investigations considering also, e.g., restrictions on $I_{\text {off }}$ or fabrication-related issues.

\section{Acknowledgment}

Financial support by Fujitsu Laboratories Ltd (Akiruno, Japan) is gratefully acknowledged.

\section{References}

[1] Pfeiffer U and Röwer T 2003 Phys. J. 229

[2] Taur Y et al 1997 Proc. IEEE 85486

[3] Kedzierski J et al 2001 Tech. Dig. Int. Electron Devices Meeting (Washington, DC) pp 437-40 
[4] Rim K et al 2001 Dig. of the Symp. on VLSI Technology (Kyoto 2001) pp 59-60

[5] Bufler F M, Asahi Y, Yoshimura H, Zechner C, Schenk A and Fichtner W 2003 IEEE Trans. Electron Devices 50 418

[6] Rieger M M and Vogl P 1993 Phys. Rev. B 4814276

[7] Jacoboni C and Reggiani L 1983 Rev. Mod. Phys. 55645

[8] Sangiorgi E and Pinto M R 1992 IEEE Trans. Electron Devices 39356

[9] Ismail K, Nelson S F, Chu J O and Meyerson B S 1993 Appl. Phys. Lett. 63660

[10] Vogelsang T and Hofmann K R 1993 Appl. Phys. Lett. 63186

[11] Miyata H, Yamada T and Ferry D K 1993 Appl. Phys. Lett. 62 2661
[12] Fischetti M V and Laux S E 1996 J. Appl. Phys. 802234

[13] Bufler F M, Graf P, Keith S and Meinerzhagen B 1997 Appl. Phys. Lett. 702144

[14] Bufler F M and Fichtner W 2003 IEEE Trans. Electron Devices $\mathbf{5 0} 278$

[15] Mizuno T, Takagi S, Sugiyama N, Satake H, Kurobe A and Toriumi A 2000 IEEE Electron Device Lett. 21230

[16] Bufler F M, Keith S and Meinerzhagen B 1998 Proc. Int. Conf. on the Simulation of Semiconductor Processes and Devices (Leuven 1998) pp 239-42

[17] Lundstrom M 1997 IEEE Electron Device Lett. 18361

[18] Lochtefeld A and Antoniadis D A 2001 IEEE Electron Device Lett. 22591 